\title{
Natural Frequency based Swing up and GA tuned Pole Placement with integral Control based Stabilization of Rotary Inverted Pendulum
}

\author{
Vishwa Nath ${ }^{1}$, R. Mitra ${ }^{2}$ \\ ${ }^{I}$ (Department of Electronics and Communication Engineering, Indian Institute of Technology, Roorkee, India) \\ ${ }^{2}$ (Department of Electronics and Communication Engineering, Indian Institute of Technology, Roorkee, India)
}

\begin{abstract}
This paper aims at the applications of natural frequency of inverted pendulum and genetic algorithm (GA) tuned pole placement with integral control in the swing up and stabilization of Rotary inverted pendulum (RIP) respectively. RIP is an important multivariable system in control to test the performance of any controller due to its nonlinear, unstable, non-minimum phase and under actuated nature. The technique used in this paper for swing up of pendulum from downward position to upright position is based on natural frequency of pendulum. It can be calculated either experimentally or based on equation of time period of pendulum. Classical method only places the dominant pair of poles to desired location hoping that higher order poles do not affect the second order approximation. This disadvantage is overcome by pole placement technique which places the entire poles at desired locations. Integral action is required to remove the steady state error. In between the swing up and stabilization a mode controller is used which is basically a condition check on the angle of the pendulum rod. Finally Simulink based experimental results are used to verify and validate the performance of the above controllers on RIP system.
\end{abstract}

Keywords: Genetic algorithm, Natural frequency based swing up controller, Pole placement with integral control, RIP

\section{Introduction}

The Rotary Inverted Pendulum (RIP) system is a multivariable, unstable, complex, non-minimum, unstable system. A majority of design technique in modern control theory is based on the state feedback configuration. Classical method like conventional PID control has a simple structure, but only places the dominant pair of poles to desired location hoping that higher order poles do not affect the second order approximation. Second drawback of classical design is that if parameters of plant change the adjustable parameters are difficult to tune [1]. To overcome these drawbacks it is better to use state space design techniques. Pole Placement is one of them. This design technique places all the poles to be placed at predefined locations. If plant is type 0 system then we have to use pole placement with integrator to eliminate error [2].

Various techniques are described for swinging up of pendulum rod that is to bring the pendulum rod from down hanging stable position to the upright unstable position. But these techniques use feedback mechanism. Swing up becomes bit difficult and tricky if there appears error in the feedback mechanism may be due the improper working of sensors. Therefore, a more simple way to swing up is based on natural frequency of pendulum. It can be calculated either experimentally by measuring time period using stopwatch or by calculating the time period of the oscillation based on the pendulum rod. During swing up pendulum change its direction at every half of time period of pendulum except very first time where it changes its direction at one fourth of its time period. If we apply a changing constant control input based on time period to the arm of pendulum, we can swing up the pendulum without any feedback from RIP system.

\section{Mathematical Model Of The System}

The GoogolTech Rotary inverted pendulum (GRIP2001) is used in the experiment as shown in Fig. 1. It is driven by a Panasonic AC servo motor which is controlled through a motion controller card (GT400-SV). The horizontal rotation of the arm $(\theta)$ is actuated by an electromotor. The pendulum rod is connected to one end of the pivot arm and is free to rotate in vertical plane $(\alpha)$ and is not actuated. As the system has two degrees of freedom and only one actuator, it is called an under-actuated system [3]. 


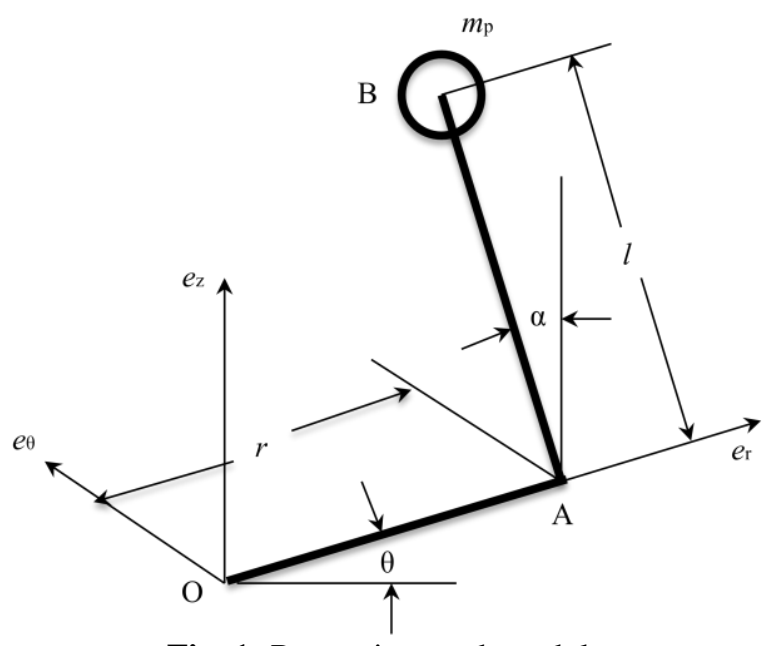

Fig. 1: Rotary inverted pendulum

Taking arm acceleration $\ddot{\theta}$ as input we get the following differential equation for RIP

$$
\frac{4}{3} m_{p} l_{p}^{2} \ddot{\alpha}-m_{p} r l_{p} \ddot{\theta}-m_{p} g l_{p} \alpha=0
$$

After simple algebraic manipulation in Eq. (1) we obtain the following state-space representation of the RIPsystem for GRIP (2001)

$$
\begin{aligned}
& {\left[\begin{array}{c}
\dot{\theta} \\
\dot{\alpha} \\
\ddot{\theta} \\
\ddot{\alpha}
\end{array}\right]=\left[\begin{array}{cccc}
0 & 0 & 1 & 0 \\
0 & 0 & 0 & 1 \\
0 & 0 & 0 & 0 \\
0 & 37.22 & 0 & 0
\end{array}\right]\left[\begin{array}{c}
\theta \\
\alpha \\
\dot{\theta} \\
\dot{\alpha}
\end{array}\right]+\left[\begin{array}{c}
0 \\
0 \\
1 \\
0.84
\end{array}\right] \ddot{\theta} } \\
& \mathrm{y}=\left[\begin{array}{c}
\theta \\
\alpha
\end{array}\right]=\left[\begin{array}{llll}
1 & 0 & 0 & 0 \\
0 & 1 & 0 & 0
\end{array}\right]\left[\begin{array}{c}
\theta \\
\alpha \\
\dot{\theta} \\
\dot{\alpha}
\end{array}\right]+\left[\begin{array}{l}
0 \\
0
\end{array}\right] \ddot{\theta}
\end{aligned}
$$

where $\theta, \alpha, \dot{\theta}$ and $\dot{\alpha}$ are the state variable of the plant.

\section{Natural Frequency Based Swing Up Controller}

The swing-up controller swings the pendulum from the initial vertically downward position to vertically upward position. If pendulum has a length of $2 \mathrm{l}_{\mathrm{p}}$ and its centre of mass is located at its geometric centre. Where $l_{p}$ is the length between rotating and mass centre of pendulum rod as shown in figure 2 . Then natural frequency for small oscillations of the pendulum is given by

$$
f=\frac{1}{2 \pi} \sqrt{\frac{m g l_{p}}{I_{p}}}
$$

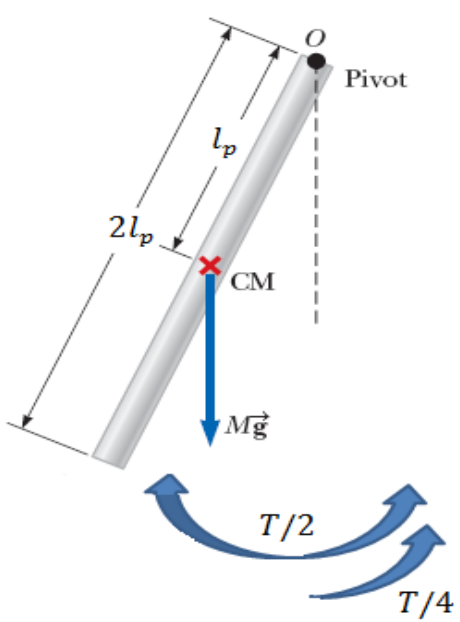

Fig. 2: Rigid Pendulum

where $\mathbf{I}_{\mathbf{p}}=\frac{\mathbf{1}}{2 \pi} \sqrt{\frac{\mathbf{m g l}_{\mathbf{p}}}{\mathbf{I}_{\mathbf{p}}}}$ moment of inertia of pendulum about one end. Putting this value in Eq. (4) we will get 


$$
\begin{gathered}
\mathrm{f}=\frac{1}{2 \pi} \sqrt{\frac{3 \mathrm{~g}}{4 \mathrm{l}_{\mathrm{p}}}} \\
\mathrm{T}=\frac{1}{\mathrm{f}}
\end{gathered}
$$

where $\mathrm{T}$ is time period of oscillation which is fixed for individual pendulum. We can find the time period using above formula or experimentally. In experimentally we apply an impulse at pendulum and measure the time period. Based on this time period we can decide the direction in which we move the arm to feed the energy to the plant for the swing up. Time period of GRIP (2001) pendulum is $1.23 \mathrm{sec}$.

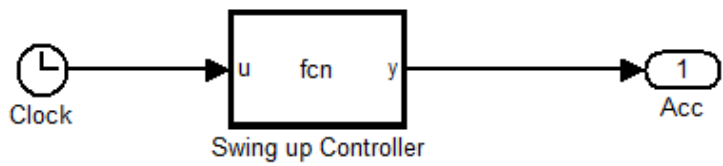

Fig. 3: Implementation of natural frequency based swing-up controller in Simulink

In this method no feedback from plant is required and decision is based on the time which is given by the clock in Simulink. Starting from the rest pendulum first changes its direction at T/4 second and after that every $\mathrm{T} / 2$ second direction change repeatedly. So using the clock we decide at which time reverse the direction of movement of arm to feed the energy in the plant for swing up the pendulum.

\section{Pole Placement With Integral Control}

Pole placement controller is not able to eliminate error if plant has no integrator (type 0 plants) because it feed only proportional and derivative of state variable we want to control. The basic principle of design of type 1 servo system is to insert an integrator in the feed forward path between the error comparator and the plant as shown in Fig. 4 [1].

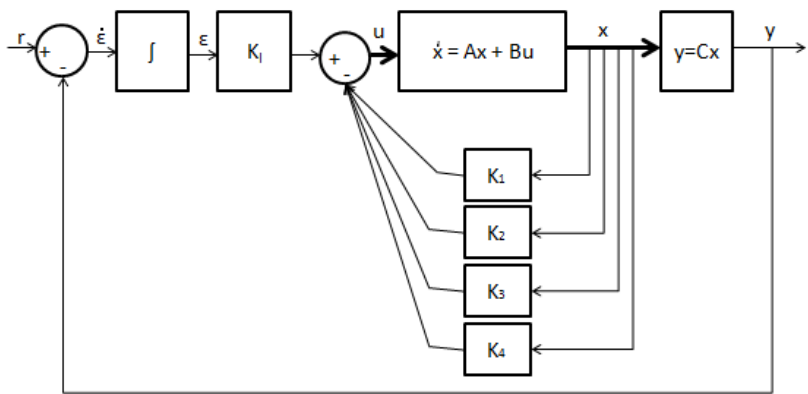

From Fig. 4 we obtain

Fig. 4: Block Diagram of pole placement controller with integrator

$$
\dot{\varepsilon}=\mathbf{r}-\mathbf{y}=\mathbf{r}-\mathbf{C x}
$$

where $\mathbf{x}=$ state vector of the plant and $\mathrm{r}$ is reference input. Using the new state variable $\boldsymbol{\varepsilon}$, new state matrix of the plant is given as,

$$
\mathbf{A}_{\text {hat }}=\left[\begin{array}{cc}
\mathbf{A} & \mathbf{0} \\
-\mathbf{C} & \mathbf{0}
\end{array}\right] \text { and } \mathbf{B}_{\text {hat }}=\left[\begin{array}{l}
\mathbf{B} \\
\mathbf{0}
\end{array}\right]
$$

Here now represent control signal $u$ as a linear combination of the state variables and new state variable

$$
\mathbf{u}=-\mathbf{K x}+\mathbf{K}_{\mathbf{I}} \varepsilon
$$

and new state feedback gain matrix

$$
\mathbf{K}_{\text {hat }}=\left[\begin{array}{ll}
\mathbf{K} & -\mathrm{K}_{\mathrm{I}}
\end{array}\right]
$$

\section{Genetic Algorithm Tuned Pole Placement With Integral Control}

Genetic algorithms are global, parallel search and optimization methods founded on Darwinian principles. This works with a population of potential solutions to a problem. The population is evolved over generations to get the best possible solution [6]. Fig. 5 shows the flow chart of GA to search the feedback matrix gain which satisfies our requirement. In this algorithm first we create the initial population and check that our 
requirement is satisfied or not. In case it is not satisfied every time a new population is created by using few percent of champions and rest is from crossover of parents.

Fitness calculation is based on match method. The calculation is designed to find a $\mathrm{K}$ vector that will drive the state space model to within $5 \%$ of both user provided criteria. In order to match both criteria, a score is given to each (overshoot and settling time) and the maximum between those is given to the genome.

$$
\begin{aligned}
& \max \left(\left|\frac{\text { maximum overshoot }- \text { desired overshoot }}{\text { desired overshoot } * 0.05}\right|\right. \text {, } \\
& \text { fitness } \left.=\left|\frac{\text { measured settling time }- \text { desired settling time }}{\text { desired settling time } * 0.05}\right|\right)+10 * \text { measured undershoot }
\end{aligned}
$$

If fitness value is feasible, feedback gain matrix is added to population along with fitness value otherwise discarded. Once the initial population is created it is sorted best on fitness value in increasing order. If the lowest fitness value satisfy user criteria the process is stop otherwise new generation is created again [8].

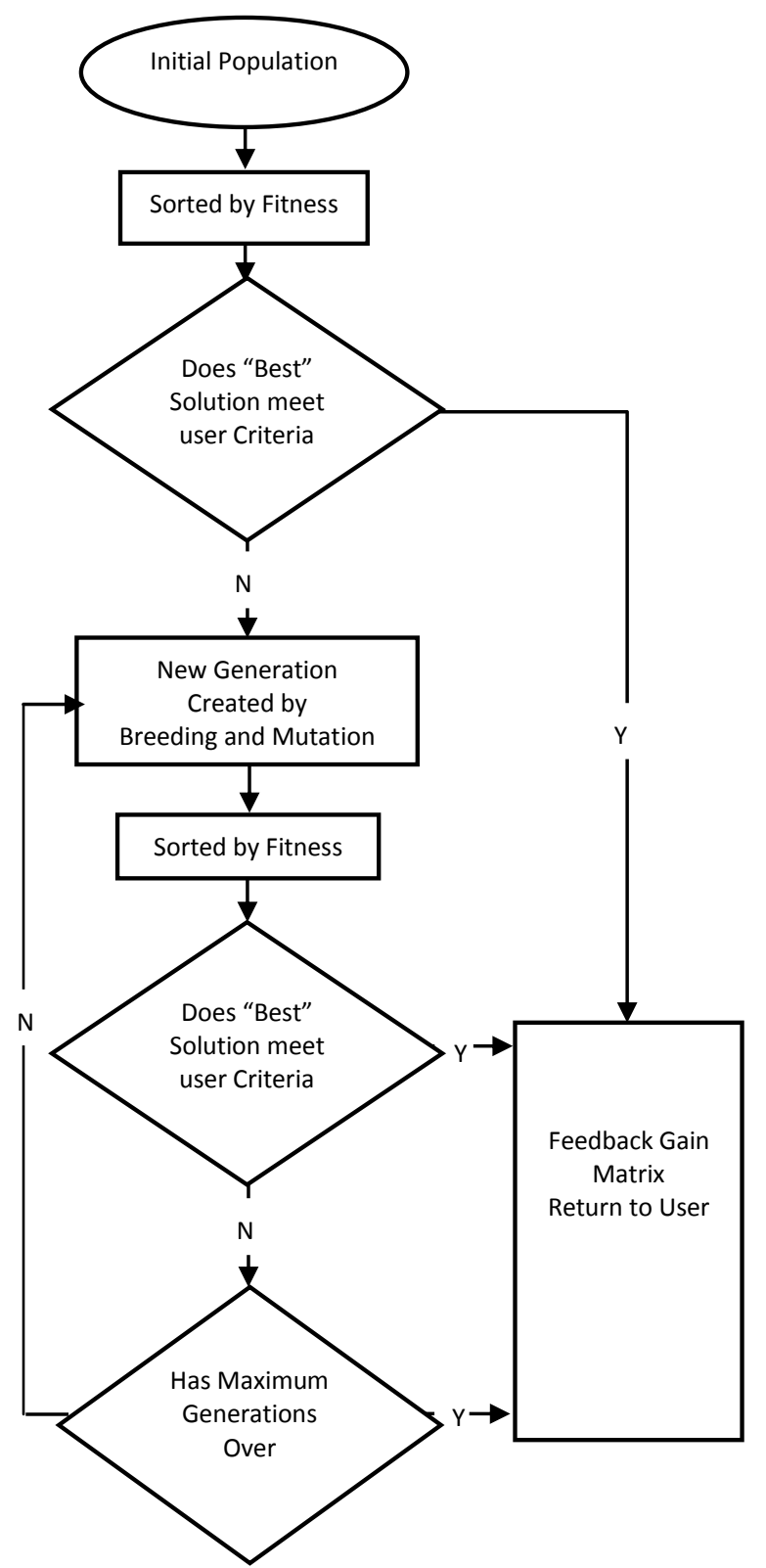

Fig. 5: Flow chart of GA for Pole Placement with integral Controller 


\section{Experimental Results}

Fig. 6 shows the Simulink model of complete experimental control system for RIP (GRIP2001). Block $-p i \sim p i$ is used to change input signal between $-p i \sim p i$ in $2 * p i$ period. In real control there are two API modules "Set Cart's Acc and Vel" and "Get Pend's Angle". These modules are used to set the arm acceleration and get pendulum and arm position also respectively [2]. Googoltech motion controller card (GT400-SV) provides API Module "Set Cart's Acc and Vel" and the same is taken as input to set the velocity and acceleration of rotating arm.

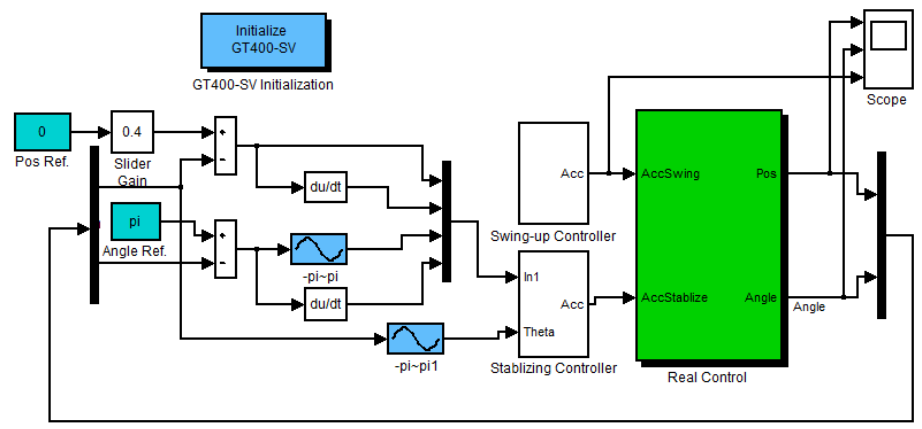

Fig. 6: Experimental Simulink model of complete control system for (GRIP2001)

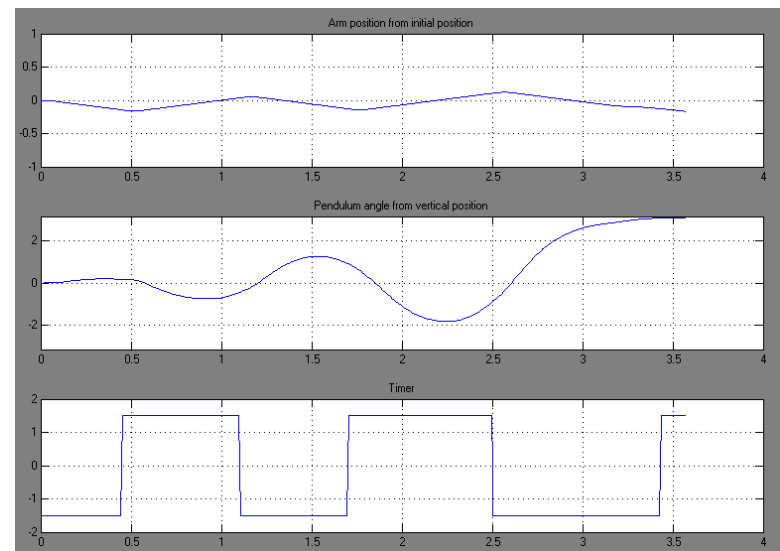

Fig. 7: Arm position and pendulum angle during swing-up using natural frequency based technique

Experimental result in Fig. 7 shows that swing-up time is nearly $3.5 \mathrm{sec}$. We can reduce it further by increasing the gain of acceleration taken as input for plant. There is a switch for switching in between the swingup and stabilizing controller according to the angle of pendulum from vertical position in real control block. In this experiment it is taken as 20 degree. Using dominant pole concept, we calculate the location of desired poles for $2 \%$ overshoot and 2 second settling time

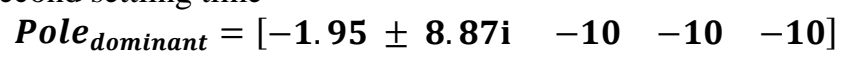

Using these pole locations along with the Ackermann's formulas, we calculate the feedback gain matrix $\mathrm{K}_{\text {hat }}$

$$
K_{\text {hat }}=\left[\begin{array}{lllll}
-769.50 & 1556.90 & -184.40 & 260.10 & 2214.70
\end{array}\right]
$$

Using GA tuned pole placement with integral control, we compute the location of desired poles for $2 \%$ overshoot and 2 second settling time

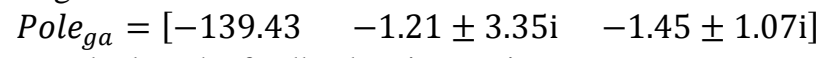

Using these pole locations we calculate the feedback gain matrix $\mathrm{K}_{\text {ga }}$

$$
K_{g a}=\left[\begin{array}{lllll}
-165.39 & 1261.77 & -82.69 & 206.27 & 140.27
\end{array}\right]
$$

Taking a particular state $\mathrm{x}=[1 ; 1 ; 1 ; 1 ; 1]$ we can calculate the control signals

$$
u_{\text {dominant }}=-3077.8 \text { and } u_{g a}=-1333.2
$$

We can see that very less control signal effort is required in case of genetic algorithm tuned pole placement with integral control in compare to dominant pole based pole placement with integral control. 
During steady state arm position is at set initial position and pendulum is at vertical upright position as shown in Fig. 8 and Fig. 9 respectively. During vertical upright pendulum angle is maintained at equilibrium point ( $\alpha=0$ radian) with a $2 \%$ error band as shown in Fig. 9.

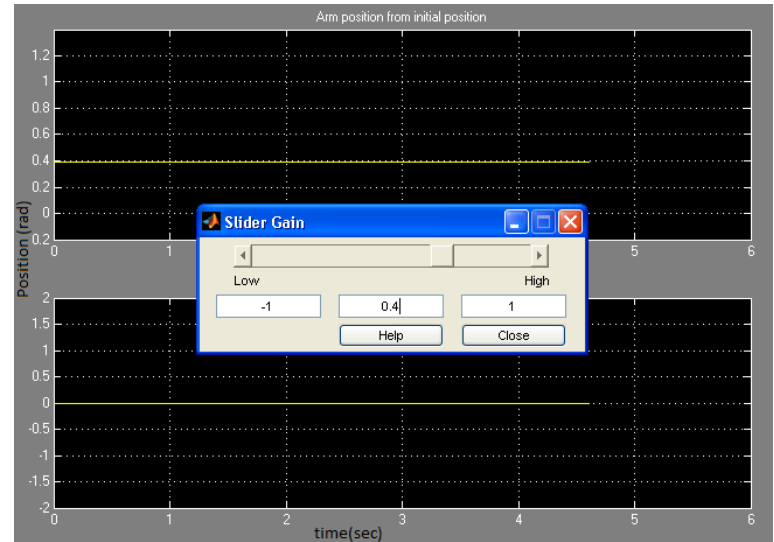

Fig. 8: steady state response of arm position for input 0.4 radian

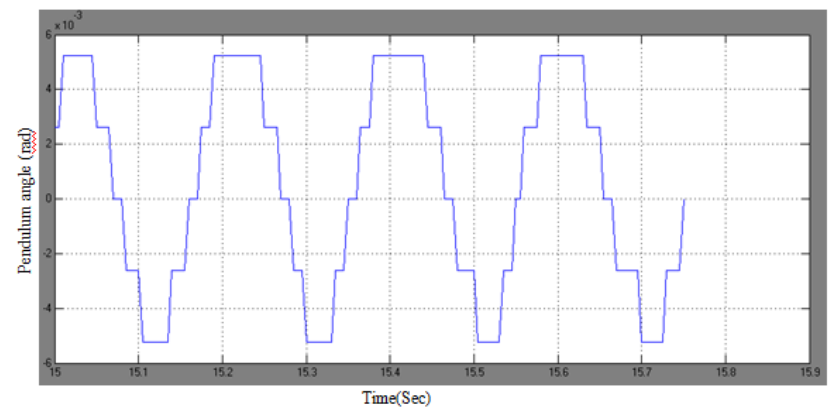

Fig. 9: Pendulum angle with vertical axis during steady state

\section{Conclusion}

Natural frequency based swing up is an open loop swing up technique and it can swing up the pendulum without any feedback from encoder mounted on it. An experimental result for swing up of pendulum using natural frequency based technique is nearly 3.5 second shown as in Figure 6. We can reduce it further by increasing the gain of acceleration taken as input for plant. Arm position is varying within 0.2 radian during swing-up.

The main advantages of genetic algorithm tuned pole placement with integral control, it consider the effect of zero in transient response and less control effort is required to get the desired response. Integral action eliminates the error in type 0 system.

\section{Books:}

\section{References}

[1]. M. Gopal, Digital Control and State Variable Method, Tata McGraw Hill, NJ, 2011, 3th Ed., pp. 393.

[2]. K. Ogata, Modern Control Engineering, PHI Learning Private Lltd, Rimjhim House, NJ, 1997, 5th Ed., pp. 70-72 and pp. 739-950.

[3]. Googoltech, Experiment Guidebook of GCIP V1.1 1209, 2009, pp.36-37,www.googoltech.com

[4]. QUANSER INNOVATE EDUCATE SRV02-Series Rotary Experiment \#7

[5]. B. C. Kuo, Automatic Control Systems, Prentice-Hall, Englewood Cliffs, NJ, 1995, 7th Ed., pp. 179 and pp. 221.

[6]. S. N. Sivanandam and S. N. Deepa, "Introduction to Genetic Algorithms", Springer, ISBN 978-3-540-73189-4, SPIN: 12053230

\section{Journal Papers:}

[7]. P. J. Fleming and R. C. Purshouse, "Genetic Algorithm in Control Systems Engineering", Control System, Robots And Automation- Vol. XVII- Genetic Algorithms in Control System Engineering

[8]. Mohd Sazli Saad, Hishamuddin Jamaluddin and Intan Zaurah Mat Darus, " Implementation of PID Controller tunning using Differential Evolution and Genetic Algorithms", International Journal of Innovative Computing, Information and Control, Volume 8, Number 11, November 2012

[9]. Vishwa Nath, R. Mitra, "Genetic Algorithm Tuned Pole Placement with Integral Control", International Journal of Scientific Research Engineering \& Technology (IJSRET), ISSN 2278 - 0882 Volume 3 Issue 2, May 2014

[10]. Y. J. Cao and Q. H. Wu, “Teaching Genetic Algorithm using MatLab”, Int. J. Elect. Enging. Educ., Vol. 36, pp. $139-153$. Manchester U.P., 1999. Printed in Great Britain 